\title{
Weather Hazard Risk Quantification for sUAS Safety Risk Management
}

\author{
CHristopher A. Roseman AND Brian M. ARgrow \\ University of Colorado Boulder, Boulder, Colorado
}

(Manuscript received 23 January 2020, in final form 1 June 2020)

\begin{abstract}
As the number of applications for small unmanned (i.e., remotely operated) aircraft systems (sUAS) continues to grow, comprehensive safety risk assessment studies are required to ensure their safe integration into the National Airspace System. One source of hazards for sUAS that has not been extensively addressed is adverse weather. A framework is presented for analyzing weather forecast data to provide sUAS operators with risk assessment information that they can use for making risk-aware decisions. The sUAS Weather Risk Model (sWRM) framework quantifies weather hazard risk for sUAS operations in rural to urban environments using weather forecast, population density, structure density, and sUAS data. sWRM is developed by following the safety risk management guidelines from the U.S. Federal Aviation Administration. Development of sWRM highlights a number of aerospace and meteorological research areas that must be addressed prior to weather risk models for sUAS becoming operational. Primary among these research areas is developing widely available finescale $(<1 \mathrm{~km})$ weather forecasts and conducting extensive sUAS flightreport studies to accurately estimate parameters of Bayesian belief network conditional probability tables used in the proposed framework. As a proof of concept, sWRM was applied over Boulder, Colorado, using the High-Resolution Rapid Refresh weather product. This initial demonstration of sWRM highlights the potential effectiveness of a detailed risk assessment model that takes into account high-resolution weather and environmental data.
\end{abstract}

\section{Introduction}

Unmanned (remotely operated) aircraft system (UAS) technology continues to expand into new markets and will soon be ubiquitous in the U.S. National Airspace System (NAS). In April 2019, the U.S. Federal Aviation Administration (FAA) approved the first air carrier delivery service for limited operations in the United States, marking the beginning of the introduction of commercial UAS into the U.S. NAS (Martin 2019). The FAA predicts that there could be as many as 4 million commercial and hobby small UAS (sUAS) flying in the United States by 2022 (Lukacs and Bhadra 2017). Commercial and research applications of sUAS include atmospheric measurements (Elston et al. 2011; Bonin et al. 2013; Elston et al. 2015), precision agriculture (Hunt et al. 2010; Zhang et al. 2012; Geipel et al. 2014), disaster response and recovery (Tuna et al. 2014; Erdelj et al. 2017), package delivery (Mathew et al. 2015; Murray and Chu 2015; Thiels et al. 2015), video surveillance (Haddal and Gertler 2010), and more. Many of these

Corresponding author: Christopher A. Roseman, christopher. roseman@colorado.edu
sUAS operations will take place over moderately and densely populated areas, posing a significant safety risk to both people and property. The FAA and NASA commissioned the UAS Traffic Management (UTM) research program to investigate how sUAS can be safely integrated into the NAS (Bradford 2018). As is the case with Air Traffic Control for in situ piloted aircraft, a critical part of the UTM system will be the analysis and quantification of risk. Comprehensive safety risk management (SRM) studies must be conducted to support the proper development of the UTM system. One source of safety hazards that has always plagued the aerospace industry, and that will certainly have a big impact on the sUAS industry, is adverse weather (Ranquist et al. 2017; Cornman and Chan 2017; Campbell et al. 2017a,b).

A 2010 FAA study of the airline industry showed that weather was a cause or contributing factor for about $20 \%$ of all accidents between 2003 and 2007 (Aviation Safety Information Analysis and Sharing 2010). Data in that report were mostly from accident reports of piloted aircraft and large, military-style UAS, with little data coming from sUAS operations. Despite a lack of accident data for sUAS, it is obvious that their relatively small size and weight make them more sensitive to 
adverse weather than are piloted aircraft or large UAS. Ranquist et al. (2017) detailed the impacts that weather can have on sUAS operations that include the following examples: unexpected wind gusts can displace unmanned (remotely operated) aircraft (UA) from their flight paths, leading to a loss of control or collision with nearby structures; turbulence can reduce the controllability of an sUAS or increase the power required to stabilize the UA; sun glare can inhibit visibility, causing an sUAS that requires line of sight to fly unobserved for a period of time; and precipitation can reduce the aerodynamic performance of aircraft. The weather hazards posed to sUAS are exacerbated by the fact that most of these operations take place in the atmospheric boundary layer (ABL) where weather can be highly variable and difficult to predict (Baklanov et al. 2011; Holtslag et al. 2013; Barlow 2014; Mahrt 2014). Furthermore, surface features such as buildings, trees, and hills can cause small-scale turbulence and wind gusts that are not captured by conventional weather forecasts but are important for sUAS operations. The risks to sUAS posed by adverse weather are numerous and profound.

To date, few regulations pertain to the operation of sUAS in adverse weather conditions. Current FAA regulations for sUAS operations are outlined in Part 107 of Title 14 of the Code of Federal Regulations (referred to as "Part 107") (Duncan 2016). These regulations apply to all UA weighing less than $55 \mathrm{lb}(25 \mathrm{~kg})$. Part 107 rules that might be impacted by weather phenomena include the following:

- UA must remain within visual line of site (VLOS) of the sUAS operator,

- the maximum ground speed of an sUAS is limited to $100 \mathrm{mi} \mathrm{h}^{-1}\left(87 \mathrm{kt} ; 45 \mathrm{~m} \mathrm{~s}^{-1}\right)$,

- UA must fly below $400 \mathrm{ft}(122 \mathrm{~m})$ above ground level (AGL) or within $400 \mathrm{ft}$ of structures,

- weather visibility must be no less than 3 statute miles $(4.8 \mathrm{~km})$ from the ground control station, and

- UA must be at least $500 \mathrm{ft}(152 \mathrm{~m})$ below and $2200 \mathrm{ft}$ (671 m) horizontally from clouds.

Assuming that an sUAS is flying under the above conditions, there are still significant weather hazards that can be encountered including sun glare, wind, turbulence, gusts, extreme temperature, extreme humidity, and solar storms (Ranquist et al. 2017). With the rapid increase in demand for sUAS access to the NAS, it is expected that many of these restrictions will be relaxed by the FAA, increasing the likelihood of sUAS operations in adverse weather conditions. To ensure safe flight under the current and future regulations, risks from weather hazards must be studied and quantified.
The FAA outlines an SRM process in the safety management system manual (Bristol 2019). The manual describes the five steps of the "DIAAT" process: describing the system, identifying hazards, then analyzing, assessing, and treating the risk. Describing the system involves outlining the scope of the risk analysis being performed. After the system is described, hazards within the risk assessment scope must be identified. A hazard is a condition that could foreseeably cause or contribute to an accident. Analyzing hazard risk is accomplished by identifying possible outcomes of each hazard, quantifying outcome severity, and quantifying the likelihood of each outcome. Severity is the consequence of a hazard's outcome measured in terms of the degree of loss or harm. The characterization of each severity level for UAS operations is shown in Table 1 (Bristol 2019). The 2019 SRM guidelines categorize severity only in terms of the effect on persons (both bystanders and members of the flight crew). Also shown in Table 1 are the 2012 guidelines, which include damage to the UA in its severity categorization (Huerta 2012). A severity category proposed by the authors is also presented in the table, with the proposed dollar amounts based on current accident reporting requirements (Duncan 2016). Likelihood is the probability of a hazard's outcome occurring and is rated on a scale from $\mathrm{A}$ to $\mathrm{E}$, with $\mathrm{A}$ being frequent and $\mathrm{E}$ being extremely improbable. The precise characterization of likelihood categories may vary depending on the outcome being considered as well as the type and quality of the data that are available for calculating likelihood. With sufficient data, or an appropriate system model, precise numerical cutoffs can be used to categorize likelihood. Otherwise, subjective intuition from subject matter experts may be used to determine the likelihood of a given event. After evaluating the severity and likelihood of each hazard outcome, the risk is assessed as acceptable (low), acceptable with mitigation (medium), or unacceptable (high). The risk level is assessed using a risk matrix. Different risk matrices may be used depending on the risks that can be undertaken by different organizations or operations. Figure 1 shows the risk matrix used for this study (Bristol 2019). The risk level of the system at any instance is taken to be the highest risk of all possible hazard outcomes.

Several previous studies investigated safety risk analysis for sUAS operations (Öztekin and Luxhøj 2008; Luxhøj and Öztekin 2009; Luxhøj 2013, 2015; Wackwitz and Boedecker 2015; Ancel et al. 2017; Barr et al. 2017; Belcastro et al. 2017; Krishnakumar et al. 2017; Washington et al. 2017b; Breunig et al. 2018; Denney et al. 2018; Lundby et al. 2019). Wackwitz and Boedecker (2015) outlined and demonstrated best practices for performing a hazard analysis for sUAS. In addition to 


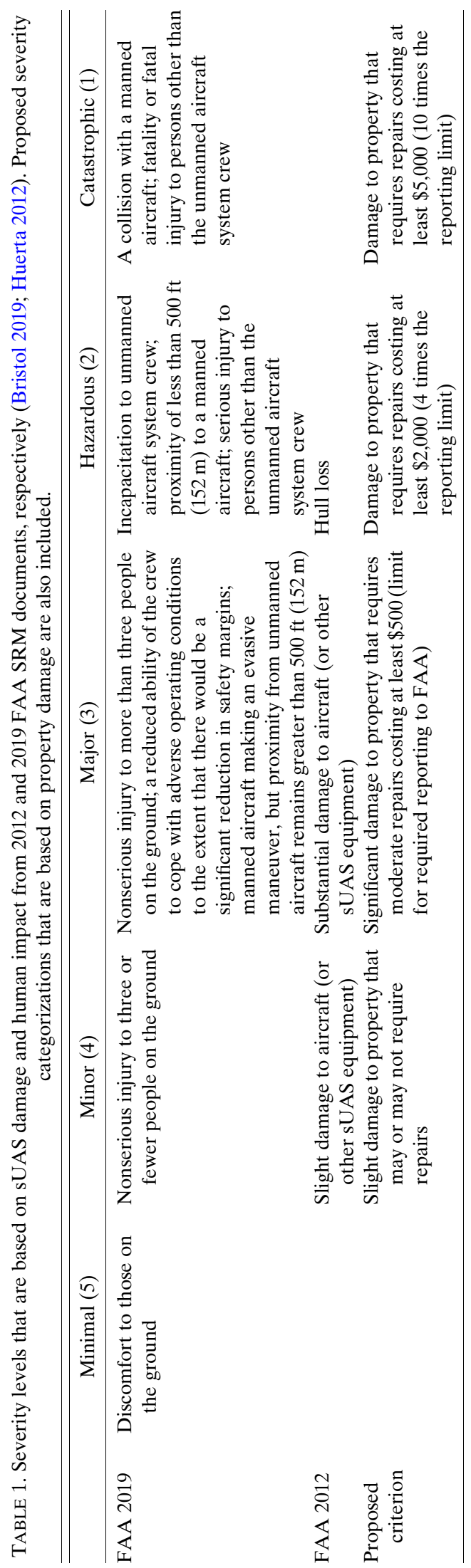

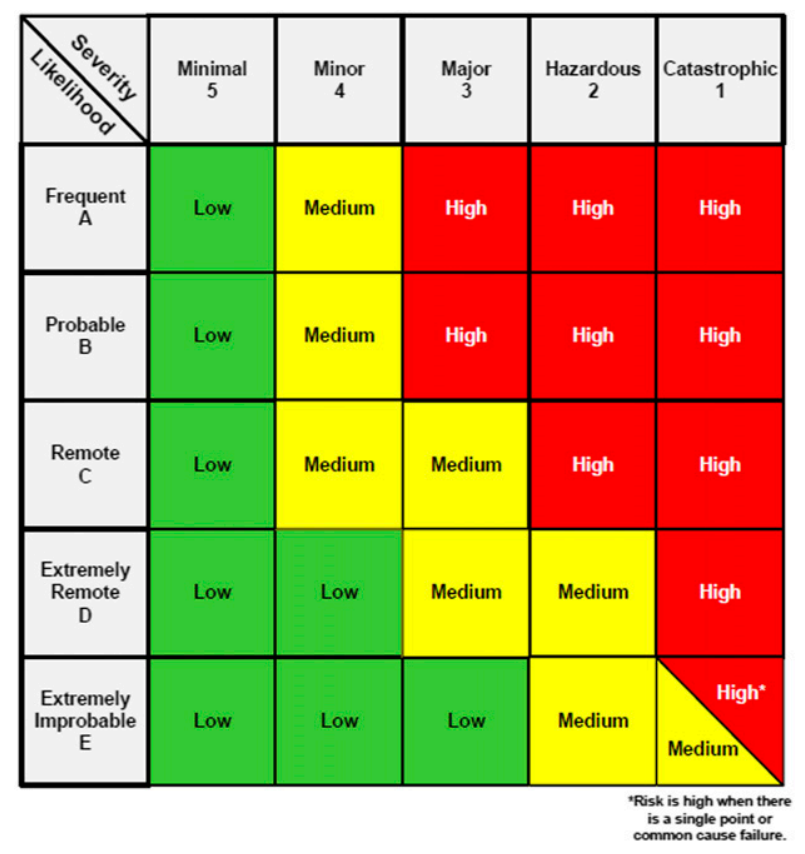

FIG. 1. Risk matrix as presented by Bristol (2019).

following the DIAAT process, they demonstrated root cause analysis and corrective action identification in order to mitigate hazard effects. Primatesta et al. (2020) developed a risk model that produces a two-dimensional map showing the probability of different accidents. Their goal was to quantify risk to the population on the ground by accounting for sUAS properties, structure data, population data, coverings over people, and wind. Barr et al. (2017) performed a preliminary risk assessment of sUAS operations that focused on calculating the risk while varying UA weight and configuration (multirotor, fixed wing, or remotely operated helicopter), population density (from remote to congested), and operational complexity [from single VLOS sUAS to multiple beyond VLOS (BVLOS) sUAS]. They used two approaches to evaluate risk. Their first approach used the DIAAT process with subjective intuition for determining likelihood. The second approach used a Bayesian belief network (BBN) model of the sUAS to calculate hazard outcome likelihood. Ancel et al. (2017) used a BBN model to make real-time estimations of risk during an sUAS operation. A number of other studies have used BBNs for quantifying risk to sUAS (Öztekin and Luxhøj 2008; Luxhøj and Öztekin 2009; Luxhøj 2015; Washington et al. 2017b; Denney et al. 2018; Washington et al. 2019).

Bayesian belief networks are commonly used for risk assessment and decision making with uncertainty. A brief description of BBNs is given here; Fenton and Neil (2013) provides a more thorough presentation. A BBN 
is a system model that consists of nodes connected in an acyclic graph. Each node represents a specific event that can occur in the system. These nodes can have a number of mutually exclusive outcomes. Root or evidence nodes are those with no parents and are defined by prior probabilities that come from prior knowledge about the system being modeled. Child nodes are defined by conditional probability tables (CPTs) that relate the probability of a node outcome to the outcomes of that node's parents. The values used in CPTs can come from subject matter experts or data using machine learning.

Few SRM studies for sUAS conducted to date have considered the impact of weather on sUAS operations. Most studies either assume that the UA is flying in calm conditions or consider a single input of either the likelihood or intensity of wind/gusts in the system model for assessing risk. Barr et al. (2017) input a scalar value for the likelihood that wind/gusts are present. Ancel et al. (2017) used a low-order model of wind speed in the $\mathrm{ABL}$ and preloaded wind data where the wind impacted the risk calculation in how it affected UA controllability, kinetic energy, and trajectory in the event of a crash landing. As previously highlighted, there are many weather types in addition to wind or gusts that can negatively impact the safety of sUAS operations (Ranquist et al. 2017; Campbell et al. 2017a,b). As of this publication, Lundby et al. (2019) have conducted the most thorough sUAS safety study considering the impact of adverse weather. Rather than assessing risk on a low-to-high scale as discussed by the FAA, their approach results in a go/no-go output for missions in specific areas. Their methodology involves defining UA-specific ranges for different weather phenomena, outside of which the sUAS cannot operate safely. All weather phenomena must lie within their respective ranges over the trajectory of the UA in order to have a "go" output; otherwise, their software produces a "no go" output. The weather phenomena ranges used in Lundby et al. (2019) are UA specific and are generally not described by sUAS manufacturers, so they must be estimated. In addition, their study did not consider the impact of weather on sUAS components aside from the UA. More comprehensive and detailed system models are necessary to predict the impact that adverse weather types have on sUAS safety. A necessary and critical component of predicting the risk due to weather is the availability of accurate weather data on a scale that is useful for sUAS operations.

Small UAS operators can obtain weather data from a number of different resources. NOAA provides several free weather forecast products that differ in domain size, temporal resolution, and spatial resolution. One of the leading weather products for research applications is the
High-Resolution Rapid Refresh (HRRR), which currently provides forecast data with a $3-\mathrm{km}$ grid spacing over the contiguous United States (CONUS) as well as Hawaii and Alaska (Benjamin et al. 2016). A forecast with 3-km resolution can be useful for quantifying sUAS risk over relatively large areas for high-level mission planning but is insufficient for detailed risk calculations of specific sUAS operations, which often have an overall footprint of less than $1 \mathrm{~km}^{2}$. Weather forecasts can also be purchased from industry providers or obtained by running in-house targeted weather simulations. WRF is a free weather simulation software that is developed and maintained by NOAA and NCAR (Skamarock et al. 2019). Several studies have demonstrated the feasibility of using WRF (or WRF with LES) to obtain targeted higher-resolution weather data for the support of sUAS operations (Roseman et al. 2019; Glasheen et al. 2019). Soon, sUAS operations in urban environments will likely require data from high-resolution LES simulations to capture the wind and turbulence details necessary for estimating risk (Lee et al. 2019; Li et al. 2008). Sensors integrated into sUAS are increasingly providing real-time in situ weather data (Elston et al. 2015; Houston et al. 2016; Koch et al. 2018). Real-time weather data could be used for dynamic calculations of operation risk, an important capability for areas where weather is highly variable and for BVLOS operations.

The current study is focused on the quantification of risk to sUAS due to adverse weather. This area of risk assessment must be addressed as these aircraft systems become ubiquitous in the NAS. The current work differs from previous research by presenting a holistic approach to risk quantification from adverse weather (National Academies of Sciences, Engineering, and Medicine 2018). In addition to wind/gusts, the risk posed by five other weather phenomena are accounted for. Negative impacts to any component of the sUAS ranging from UA control to operator health are considered. An sUAS Weather Risk Model (sWRM) framework is presented that takes into account sUAS properties, population density, structure density, and weather forecast data to estimate risk. The goal is to demonstrate a model framework that analyzes weather forecast data to provide risk assessment information to sUAS operators so they can make risk-aware decisions. Section 2 presents the development of sWRM by following the FAA SRM DIAAT process. Section 3 presents a brief demonstration of sWRM and discusses several use cases. Section 4 highlights a number of research areas that must be addressed by the aerospace and meteorological communities before operational sUAS weather risk assessment tools can be deployed. 
TABLE 2. Hazardous weather types that might be encountered during Part 107 operations or extraordinary operations.

\begin{tabular}{ll}
\hline Part 107 operations & Extraordinary operations \\
\hline Mean wind & Rain \\
Gusts & Fog/clouds \\
Wind shear & Snow \\
Turbulence & Hail \\
Extreme temperature & Lightning \\
Extreme humidity & \\
Sun glare & \\
Solar storms & \\
\hline
\end{tabular}

\section{Model framework development}

A systematic risk assessment procedure for aerospace applications is given by the FAA in the DIAAT process described above. The following sections follow each step of the DIAAT to systematically develop a general sUAS model framework for quantifying risk from adverse weather.

\section{a. Describing the system}

The system of interest includes all the components of an sUAS that might be exposed to adverse weather. This includes risks to the UA, human operators, and any supporting equipment (e.g., ground station computers or ground vehicles). Part 107 compliance is assumed, and in addition to weather hazards that directly impact the sUAS, the risk to the operational environment (e.g., buildings or bystanders) is estimated in the event of an accident. The sUAS is assumed to be operating under nominal conditions with no hazards other than weather (e.g., mechanical, electrical, or communication failures) considered. The current model is focused on battery-powered sUAS, because these are currently the most common.

\section{b. Identify hazards}

Weather types that can be hazardous to sUAS are listed in Table 2, which divides weather phenomena into those likely to be encountered during Part 107 operations and other hazardous weather types. Forecast data provide a predicted measure of these different weather hazards over a geographic area. The potential outcomes of these weather types are outlined in the next step.

\section{c. Analyze risk}

With hazardous weather types identified, the next step in the SRM process is to identify possible hazard outcomes. Here, outcomes are divided into intermediate hazard effects and weather hazard outcomes. Intermediate hazard effects are those that might have a deleterious effect on sUAS performance but are not serious enough to require
TABLE 3. Possible effects and outcomes of weather hazards.

\begin{tabular}{ll}
\hline \hline Intermediate hazard effects & \multicolumn{1}{c}{ Weather hazard outcomes } \\
\hline $\begin{array}{l}\text { Loss of control } \\
\text { Diminished aerodynamic } \\
\text { performance }\end{array}$ & $\begin{array}{l}\text { Collision with person } \\
\text { Collision with structure }\end{array}$ \\
$\begin{array}{l}\text { Reduced battery life } \\
\text { Reduced operator } \\
\text { effectiveness }\end{array}$ & $\begin{array}{l}\text { Collision with ground } \\
\text { Loss of communication }\end{array}$ \\
& Damage to aircraft \\
& Damage to supporting equipment \\
& Harm to operator \\
\hline
\end{tabular}

mitigation. Weather hazard outcomes are the primary risks that must be quantified as low, medium, or high. Adverse weather can either directly cause a weather hazard outcome or cause a combination of intermediate effects that lead to a weather hazard outcome. A list of the intermediate hazard effects and weather hazard outcomes considered in this study is shown in Table 3 . The "collision with person" outcome refers to the UA impacting any person not directly involved with the sUAS operation. "Collision with structure" includes any impact with a human-built structure (house, office building, etc.). "Collision with ground" refers to the aircraft impacting the ground. "Damage to aircraft" refers to any damage to the UA, whereas "damage to supporting equipment" encompasses any damage sustained by hardware or software that supports UA flight. "Harm to operator" refers to any injury to persons directly involved in the operation of the sUAS. Future development of this framework could include more weather types and intermediate effects and could further subdivide weather hazard outcomes.

After identifying possible weather hazard outcomes, the severity and likelihood of these outcomes must be quantified. This calculation is a function of the weather, sUAS properties, and the operation environment. Important sUAS and environment properties that are relevant when calculating risk and likelihood are shown in Tables 4 and 5, respectively. Properties of sUAS that are important for predicting operation risk can be either general properties of the system or mission specific, leading to two different weather risk assessment approaches.

The first approach only considers general sUAS properties. With gridded forecast data over a given area, risk is evaluated at each forecast data point using other local environment data (population density, building density, etc.) and general UA data (cruise speed, maximum speed, disturbance rejection, etc.). This results in a spatial map over a specific geographic area showing where and when the risk is high, medium, or low. The second approach considers a specific sUAS flight path. The flight path includes a starting time, waypoints, and 
TABLE 4. List of sUAS properties that may be important when calculating the severity and likelihood of different hazard outcomes.

\begin{tabular}{|c|c|}
\hline sUAS properties & Units or type \\
\hline \multicolumn{2}{|c|}{ General } \\
\hline Aircraft mass & $\mathrm{kg} ; \mathrm{lb}$ \\
\hline Aircraft configuration & Multirotor; fixed wing; helicopter \\
\hline Max airspeed & $\mathrm{m} \mathrm{s}^{-1} ; \mathrm{ft} \mathrm{s}^{-1}$ \\
\hline Cruise airspeed & $\mathrm{m} \mathrm{s}^{-1} ; \mathrm{ft} \mathrm{s}^{-1}$ \\
\hline Min airspeed & $\mathrm{ms}^{-1} ; \mathrm{ft} \mathrm{s}^{-1}$ \\
\hline Max ground speed & $\mathrm{m} \mathrm{s}^{-1} ; \mathrm{ft} \mathrm{s}^{-1}$ \\
\hline Cruise alt AGL & $\mathrm{m} ; \mathrm{ft}$ \\
\hline Automatic control & Yes/no \\
\hline Disturbance rejection & None; stock; advanced \\
\hline \multicolumn{2}{|c|}{ Mission specific } \\
\hline Velocity & $\mathrm{m} \mathrm{s}^{-1} ; \mathrm{ft} \mathrm{s}^{-1}$ \\
\hline Mission duration & $\mathrm{s} ; \min ; \mathrm{h}$ \\
\hline Battery life & $\mathrm{mA} \mathrm{h}$ \\
\hline Flight alt AGL & $\mathrm{m} ; \mathrm{ft}$ \\
\hline
\end{tabular}

air or ground speed of the UA along each flight path segment. Forecast and environment data near the planned flight path (both in time and position) could be coupled with the expected aircraft state along the flight path to estimate the risk of that specific mission. This type of risk assessment could consider properties such as UA direction relative to the wind and mission duration; factors that are not considered in the first risk assessment approach. A path-specific risk assessment would be most appropriate when very high-resolution weather data (e.g., $\sim 100 \mathrm{~m}$ or finer) are available over the flight path region. Such highresolution forecasts have been used in coordination with sUAS operations (Nolan et al. 2018; Glasheen et al. 2019; de Boer et al. 2020). However, since most readily accessible forecast datasets have a relatively coarse resolution, the current study focuses on the first approach to risk assessment where only general sUAS properties are considered.

Washington et al. (2017a) present an extensive review of models that have been used to estimate ground impact risk for sUAS. These previous models differ in the outcomes they consider, the estimation of impact kinetic energy, and the quantification of damage or injury severity. The following two sections explain the development of the severity and likelihood models in sWRM. The FAA SRM guidelines emphasize that the calculations for severity and likelihood should be independent. This means that the severity and likelihood models can run in parallel, each having the same set of inputs but performing different calculations.

\section{1) QUANTIFYING SEVERITY}

Table 3 lists the possible final negative outcomes of a weather hazard. With the exception of damage to
TABLE 5. Environment properties that may be important when calculating the severity and likelihood of different hazard outcomes.

\begin{tabular}{ll}
\hline \multicolumn{1}{c}{$\begin{array}{c}\text { Environment } \\
\text { property }\end{array}$} & \multicolumn{1}{c}{ Value } \\
\hline $\begin{array}{l}\text { Population density } \\
\text { Structure density }\end{array}$ & $\begin{array}{l}\text { Remote, rural, suburban, urban, congested } \\
\text { Remote, rural, suburban, urban, congested } \\
\text { people }\end{array}$ \\
people & Percent covered/uncovered \\
\hline
\end{tabular}

supporting equipment and harm to the operator, these hazards are largely a function of the UA kinetic energy at the time of impact. The UA kinetic energy is a function of its speed and mass. Impact speed can be a function of the total wind speed (mean wind plus gusts), the vertical distance that the UA falls, UA drag, and the UA speed when last in controlled flight. Previous studies have estimated UA impact speed in a variety of ways including: setting impact speed equal to the terminal velocity of the UA in free fall (Ancel et al. 2017; Barr et al. 2017; Arterburn et al. 2017); setting impact speed equal to 1.4 times the maximum UA speed (Dalamagkidis et al. 2008); assuming the UA follows a ballistic trajectory to the ground starting from a specified altitude and speed (La Cour-Harbo 2019; Kim 2019).

The severity model in sWRM estimates impact speed in several ways, depending on what is being impacted (person, structure, or ground) and occupation of the area where the collision occurs (from remote to congested). Collisions occur after the UA experiences a loss of control. It is assumed that the loss of control is catastrophic in the sense that the UA cannot glide to a safe location or be slowed by a parachute, although these are mitigation strategies that can be added to the current framework in future studies. For collisions with the ground, persons, and buildings in suburban to remote areas, the UA impact speed is calculated by assuming it follows a ballistic trajectory. For the worst-case scenario, the UA is assumed to follow a two-dimensional trajectory that is aligned with the total wind. (To expand this framework to include trajectory-specific risk calculations, a three-dimensional ballistic trajectory could be calculated.) The two-dimensional equations of motion are written in a reference frame relative to the total wind,

$$
m \dot{\mathbf{v}}=-m g-\left(\frac{1}{2} \rho A C_{D}\right) \mathbf{v}^{2} \hat{\mathbf{v}}
$$

where $m$ is the UA mass, $\dot{\mathbf{v}}$ the acceleration, $g$ is acceleration due to gravity, $\rho$ is the air density, $A$ is the cross-sectional area of the UA, $C_{D}$ is the constant drag coefficient, $\mathbf{v}$ is the velocity of the UA, and $\hat{\mathbf{v}}$ is a unit vector 
pointing in the direction of the UA velocity. Equation (1) is written in horizontal and vertical components as

$$
\begin{aligned}
& m \dot{u}=-\left(\frac{1}{2} \rho A C_{D}\right) u^{2} \hat{u} \quad \text { and } \\
& m \dot{v}=-m g-\left(\frac{1}{2} \rho A C_{D}\right) v^{2} \hat{v},
\end{aligned}
$$

where $\dot{u}$ and $\dot{v}$ are the horizontal and vertical acceleration components, $u$ and $v$ are the horizontal and vertical velocity components, with the sign of $\hat{u}$ and $\hat{v}$ chosen such that the drag force is in the direction opposite of the UA velocity. Equation (3) can be integrated twice to find an analytical expression for the time that it takes for the aircraft to fall a distance $h$,

$$
t=\frac{v_{T}}{g} \cosh ^{-1}\left[\exp \left(g h / v_{T}^{2}\right)\right],
$$

where $v_{T}$ is the UA terminal speed given by

$$
v_{T}=\sqrt{2 m g /\left(\rho A C_{D}\right)} .
$$

Expressions for the horizontal and vertical velocity components at time $t$ are found by integrating Eqs. (2) and (3), respectively,

$$
\begin{aligned}
& u=\frac{u_{0} m}{m+u_{0} 0.5 \rho A C_{D} t} \text { and } \\
& v=v_{T} \tanh \left(-\frac{g}{v_{T}} t\right),
\end{aligned}
$$

where $u_{0}$ is the UA cruise speed. It is assumed that the UA vertical speed is negligible at the beginning of the ballistic trajectory (when the loss of control occurs). This is reasonable for cruise but may result in significant error for other phases of flight. Assuming negligible vertical wind near the ground, the total horizontal wind magnitude $W$ is then added to the horizontal velocity component to shift this calculation into the reference frame of the ground. Vertical wind near the ground can be significant under certain conditions; information about these situations should be included in future sWRM implementations. The kinetic energy KE can then be readily calculated:

$$
\mathrm{KE}=0.5 m\left[(u+W)^{2}+v^{2}\right] .
$$

For collisions with the ground and persons, it is assumed that the UA falls a height $h$ equal to the cruise altitude (AGL) of the operation. In suburban to remote environments, it is assumed that the UA impacts buildings at an average height of $20 \mathrm{ft}(6 \mathrm{~m}$; the height of a two-story
TABLE 6. Discretization relating the Abbreviated Injury Scale (AIS) to severity level of collisions with people.

\begin{tabular}{lc}
\hline \hline Severity & AIS range \\
\hline Minimal & $<1.5$ \\
Minor & $1.5-2.5$ \\
Major & $2.5-3.5$ \\
Hazardous & $3.5-4.5$ \\
Catastrophic & $>4.5$ \\
\hline
\end{tabular}

building). This means that the trajectory calculation is performed with $h$ equal to the cruise altitude minus $20 \mathrm{ft}$. Note that no distinction is made in the model for whether the impact is with the side or roof of the building. This level of fidelity would be appropriate for trajectory-specific risk assessments with detailed building information. In urban environments, it is assumed that sUAS operations will take place between tall buildings. If a loss of control event takes place there, the UA will likely impact the side of a building and only fall a short distance after losing control. This means that an appropriate and conservative estimate for impact speed could be the cruising airspeed plus the total wind.

Models relating impact kinetic energy to human injury have been developed (Clare et al. 1975; Sturdivan et al. 2004; Bir et al. 2004; Bir and Viano 2004; Magister 2010). The blunt body criterion BC is used here (Clare et al. 1975; Bir and Viano 2004; Magister 2010),

$$
\mathrm{BC}=\ln \left(\frac{\mathrm{KE}}{k D M^{2 / 3}}\right),
$$

where $k$ is the coefficient for chest wall thickness ( $\sim 0.711$ for male; $\sim 0.593$ for female), $D$ is the characteristic impact diameter of the UA $(\mathrm{cm})$, and $M$ is the mass of the person being impacted $(\mathrm{kg})$. The BC is linearly related to the Abbreviated Injury Scale (AIS) (Sturdivan et al. 2004; Magister 2010):

$$
\text { AIS }=1.328 \times \mathrm{BC}+0.603 .
$$

Table 6 shows the mapping of AIS values onto a 1-5 scale to relate AIS to severity in sWRM. Under Part 107, the maximum weight $(55 \mathrm{lb} ; 25 \mathrm{~kg})$ and ground speed $\left(100 \mathrm{mi} \mathrm{h}^{-1} ; 45 \mathrm{~m} \mathrm{~s}^{-1}\right)$ result in a maximum kinetic energy of $18500 \mathrm{ft} l \mathrm{lb}(25 \mathrm{~kJ})$. The AIS value for a woman weighing $165 \mathrm{lb}(75 \mathrm{~kg})$ hit by a UA with this kinetic energy with an impact diameter of $20 \mathrm{~cm}$ is 6.5 . This value is considered unsurvivable, highlighting the need for risk assessment studies even for sUAS in compliance with Part 107. This injury model only considers head-on impacts with a person's chest. Operational versions of sWRM should subdivide the "collision with person" outcome into "chest-collision with person" and "head-collision 


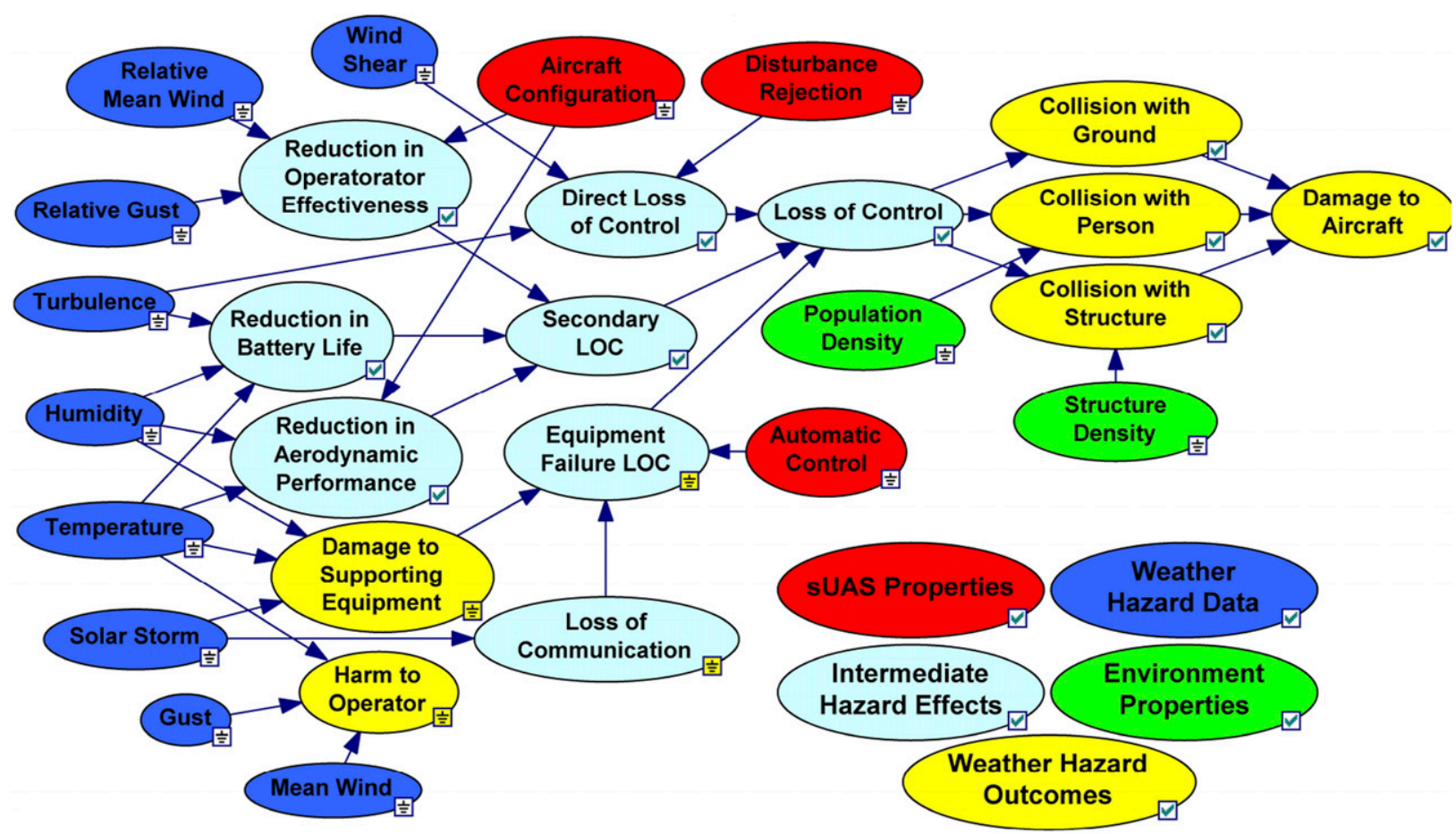

FIG. 2. Bayesian belief network used to calculate the likelihood of hazard outcomes given forecast data.

with person," calculating the likelihood and severity for each independently.

The authors are unaware of any generalized models that relate impact kinetic energy to damage severity for impacts with the ground or structures. Therefore, informed, but arbitrary kinetic energy cutoffs are used to relate impact kinetic energy to damage severity for these outcomes.

For simplicity, the severity of damage to supporting equipment and harm to operator are set to constant values of 4 (minor) and 3 (major), respectively.

\section{2) QUANTIFYING LIKELIHOOD}

As described in the introduction, several risk assessment studies have used BBNs to model sUAS and predict the likelihood of hazard outcomes. A BBN is constructed here for an sUAS exposed to several weather phenomena. Whereas earlier work only used a single node for adverse weather inputs, the sWRM BBN model employs nine nodes to more realistically predict the risk due to adverse weather types. Figure 2 shows the detailed BBN used to calculate the likelihood of hazards due to weather.

There are five different categories of nodes in the presented BBN: weather hazard data (dark blue), sUAS properties (red), environment properties (green), intermediate hazard effects (light blue), and weather hazard outcomes (yellow). The first three categories (weather hazard data, sUAS properties, and environment properties) are evidence nodes. Outcomes for weather hazard data nodes come directly from forecast data (and maximum UA speed as discussed below). Outcomes for sUAS property nodes are given by known properties of the airframe, and communications and control subsystems of the sUAS. Environment properties are known from population and structure density data. The last two node types (intermediate hazard effects and weather hazard outcomes) are child nodes whose outcomes are conditioned on their parent's outcomes. A CPT for each child node defines this conditional relationship. A description for exactly how child node outcomes are calculated can be found in Fenton and Neil (2013).

One of the difficulties in creating a generalized model is the wide range of capabilities of current sUAS; sWRM accounts for this range in two ways. First, sUAS characteristics are included as evidence nodes for the BBN (red nodes); three UA characteristics are included: UA configuration, disturbance rejection capability, and automatic control. These characteristics are included because they are the most distinguishing features of sUAS that differentiate their behavior in adverse weather. Second, diverse UA capabilities are taken into account by scaling mean wind and gusts with the maximum UA speed (relative mean wind and relative gust nodes). The reasoning for this scaling is that a typical UA has a maximum speed ranging from 3 to $40 \mathrm{~m} \mathrm{~s}^{-1}$ (Ranquist et al. 2017). A UA with a maximum airspeed of $30 \mathrm{~m} \mathrm{~s}^{-1}$ 
will be little impacted by a mean wind of $10 \mathrm{~ms}^{-1}$. However, a UA with a maximum airspeed of $5 \mathrm{~m} \mathrm{~s}^{-1}$ would be entirely overcome by the same mean wind. This example illustrates that UA respond to a relative mean wind speed scaled by the maximum airspeed. Similar logic can be applied for gusts with the caveat that, an sUAS response to gusts is also a function of the UA maximum acceleration from control actuation and propulsion. This acceleration dependence is currently not taken into account in sWRM. While the UA may respond primarily to relative mean wind and gust, the magnitude of mean wind and gusts must also be taken into account for the safety of the sUAS operators. Some UA may be able to fly in winds approaching $25 \mathrm{~m} \mathrm{~s}^{-1}$, but a sustained wind of this speed could pose a risk to the sUAS operators on the ground.

Operator effectiveness is defined as the ability of the operator or autopilot to maximally control the UA movement. As the total wind approaches the maximum speed of the UA, the UA loses the ability to traverse upwind relative to the ground. In addition, a high relative gust might displace the UA from its flight path, regardless of the reaction time of the controller.

Weather can have a major impact on UA battery capacity and performance. As turbulence intensity increases, power needed to stabilize the UA also increases, reducing the effective battery capacity available for other mission requirements. In addition, extreme humidity and temperature can be detrimental to battery life and performance (Byun et al. 2017; Shim et al. 2002). Under Part 107 restrictions, the only potential risk to aerodynamic performance is icing on the UA wings or rotor blades. The likelihood of icing is a function of temperature and humidity (Schultz and Politovich 1992).

Typical mission support equipment consists largely of electronics that can be damaged by extreme temperatures, condensation, and a highly charged atmosphere. Because Part 107 effectively requires that sUAS cannot fly near convective storms, the only major source of atmospheric charge is solar storms. Solar storms can also have a detrimental impact on GPS. Note that lightning is still possible (although unlikely) under Part 107 restrictions and should be considered as a weather hazard in future studies.

Loss of control (LOC) could be considered the most important intermediate hazard effect, as it leads to four of the six weather hazard outcomes, including the most severe outcomes. To reduce the number of CPT parameters, three independent types of LOC events are considered: direct LOC, secondary LOC, and equipment failure LOC. Direct LOC occurs when adverse weather directly impacts the UA, causing the pilot (or autopilot) to immediately lose control. Secondary LOC occurs when weather degrades different aspects of the UA (controllability, available battery capacity, aerodynamic performance) possibly leading to LOC. Equipment failure LOC occurs when adverse weather does not directly impact the UA but damages equipment necessary for UA flight (communication, measurement devices, GPS, etc.). The main LOC node then has a simple CPT (consisting of only 0 s and 1s) that effectively states that a LOC occurs if any of the previous types of LOC occur. This breakup of LOC into independent events reduces the number of CPT parameters that need to be defined from 75000 to 208. This reduction in the number of parameters greatly improves the tractability of estimating these parameters by intuition or data. The obvious downside of this method is that these different types of LOC are not independent, so the current model may not properly predict the likelihood of all possible event combinations.

After the acyclic graph is constructed, the CPTs for the child nodes are assigned. The number of independent parameters $N$ required for a given CPT is given by

$$
N=(n-1) \prod_{i=1}^{P} p_{i}
$$

where $n$ is the number of outcomes of the current node, $P$ is the number of parent nodes, and $p_{i}$ is the number of possible outcomes for the $i$ th parent node. The total number of independent CPT parameters for the BBN in Fig. 2 is 1593 . This BBN model was constructed with the goal of having the parameters of the CPT be independent of the specific sUAS. While the sWRM BBN does not fully realize this goal, it does achieve a high degree of generalization by explicitly including in the BBN the sUAS properties that are most relevant to their behavior in adverse weather. Further generalization could be accomplished by simply including more sUAS properties into the model. Some of these additional properties could include positioning technique (VLOS, single/ multiple GPS, etc.), UA characteristic length (for determining the most important turbulence scales), or maximum UA acceleration (for more accurately modeling the response to gusts). More detailed inputs such as these are not included in sWRM because they are assumed to have a much smaller effect on the differentiation between sUAS behavior than the properties that are included. Because of the generalized model construction, the 1593 CPT parameters are approximately constant for all sUAS, and do not need to be redefined by each sUAS operator. Parameters for the CPTs can be estimated from intuition or experience with the system, or machine learning with a sufficient amount of data. Unfortunately, as of this publication there is not a collection 


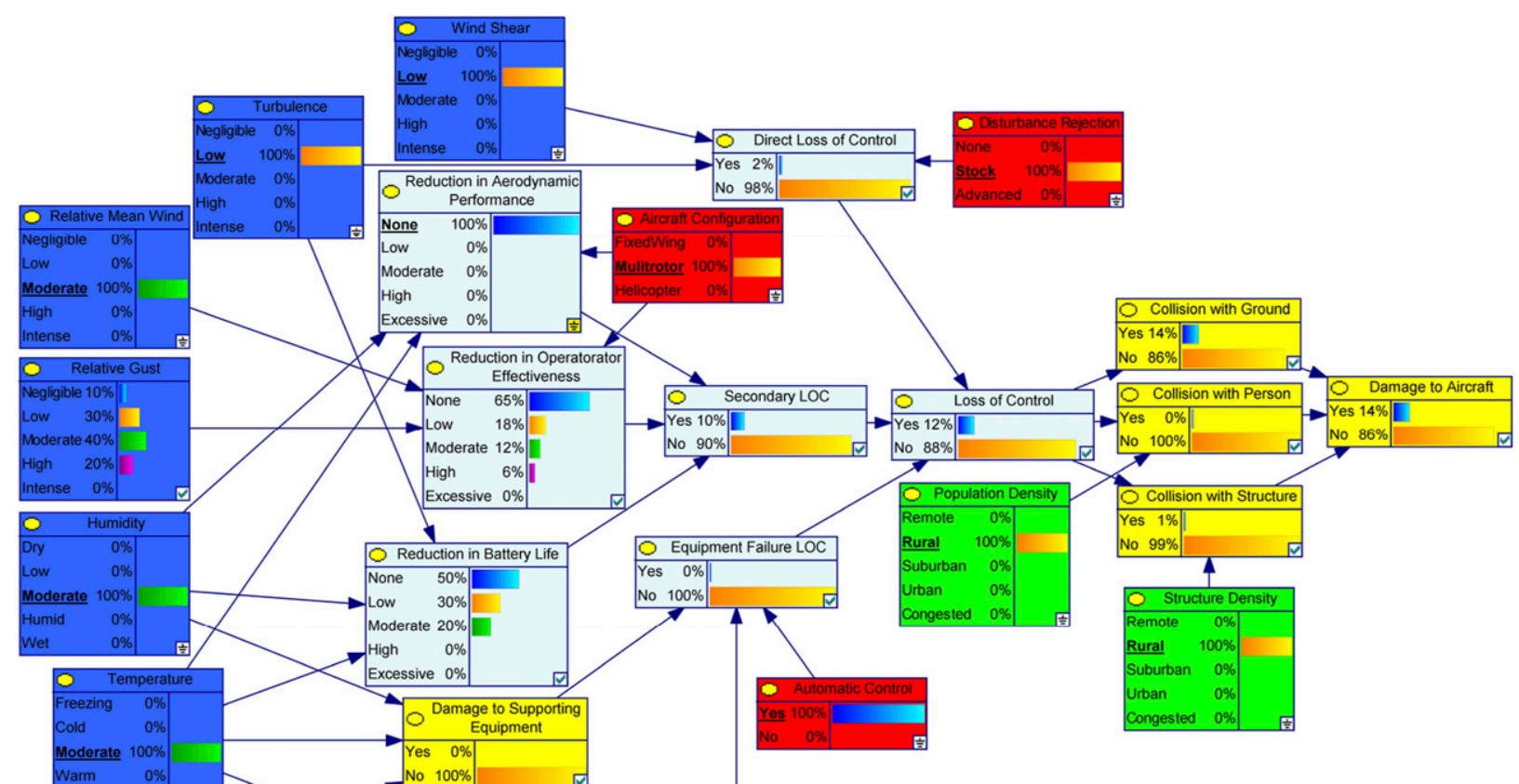

of flight-report data required to estimate the parameters with machine learning (see section 4). For the proof of concept presented below, parameters for this BBN were estimated from intuition about the behavior of the system; these estimated parameters should not be used in an operational setting.

A simple demonstration of the BBN system model for determining likelihood of weather hazard outcomes is shown in Fig. 3. This example was constructed to show the extensive functionality of the BBN framework for likelihood calculations. The bar graphs in each of the nodes help to visualize the likelihood that each of the node outcomes occur (color of the bars have no inherent meaning). It is possible that predictions for all weather input nodes may not be available. When this is the case, a distribution of likely values for unknown nodes can be used. This is demonstrated with the relative gust, solar storm, and gust nodes.

The BBN estimates the probability of different outcomes, which can be interpreted as the number of times the event is predicted to occur in a given number of operations that experience that same system state. For example, if the $\mathrm{BBN}$ calculates that the probability of colliding with a building is $1 \%$, that means that 1 in every 100 sUAS operations that experience that particular system state will collide with a building. The probabilities produced by the BBN must be related to the likelihood categories shown in the risk matrix in Fig. 1. In the FAA safety management system manual (Bristol 2019), precise numerical definitions are given for the likelihood categories. The CPT parameters estimated by the authors appear to be highly conservative relative to the likelihood categories from the FAA. For the purposes of the model proof of concept shown below, the likelihood categories were adjusted in order to produce interesting results but are not meaningful in a practical sense. As mentioned above and in section 4, further research is required to more accurately estimate the CPT parameters of the BBN.

\section{d. Assess risk}

The models outlined above predict outcome severity on a scale from minimal (5) to catastrophic (1) and 


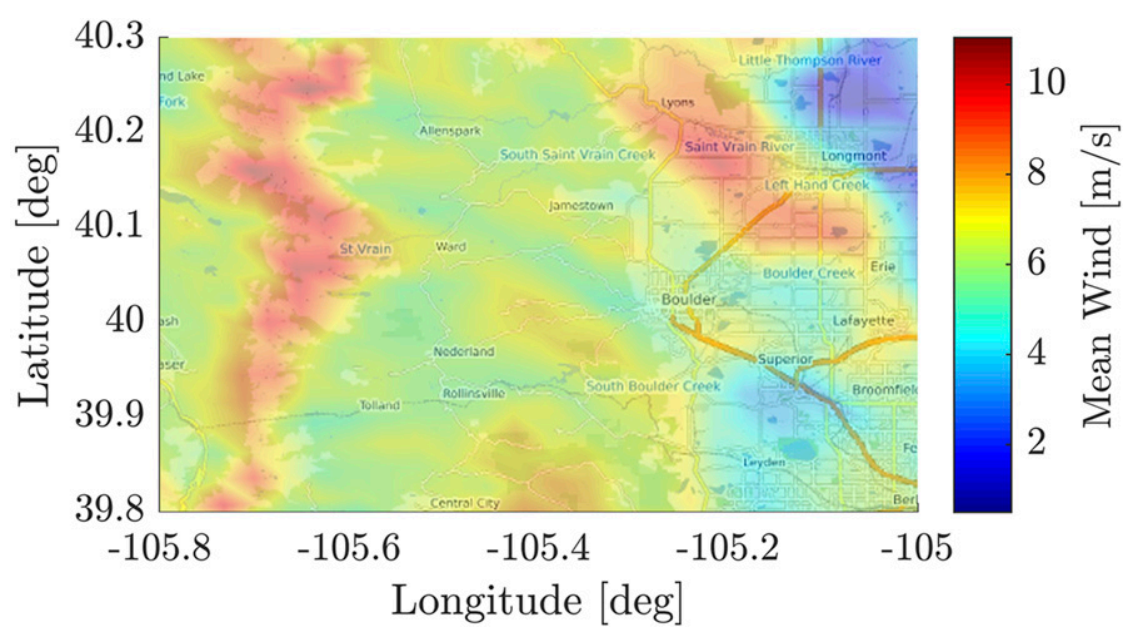

FIG. 4. Mean wind over Boulder County from HRRR forecast.

predict likelihood on a scale from frequent (A) to extremely improbable (E). Assessing the risk consists of plotting severity and likelihood values in a risk matrix, with risk labeled as low, medium, or high. The risk matrix used in this study is shown in Fig. 1, following the method of Bristol (2019).

\section{e. Treat risk}

Treating or mitigating the risk is the procedure to reduce the risk to an acceptable level. For situations where the risk level exceeds the acceptable value during the execution of a flight, the system must dynamically respond in real time to mitigate that risk. The current study is focused on quantifying the risk of planned operations before they begin. Real-time risk assessment technology is discussed in section 3. Options for mitigating risks in a planned operation are to

1) modify the sUAS to be more robust to adverse weather,

2) adjust the flight path to avoid high risk areas,

3) move the original flight time to before or after an adverse weather event, or

4) cancel the proposed flight.

These treatment options could be performed manually after a risk assessment is performed or could be accomplished by high-level automated planning algorithms. Intricate path-planning and decision-making methods could be used to plan optimal flight paths that take into account the acceptable risk of a given sUAS as has been previously demonstrated (Guglieri et al. 2015; Castagno et al. 2018; Primatesta et al. 2019,2020). The details of how to adjust the proposed flight plan are highly dependent on the specific operation and therefore are not included in sWRM.

\section{Proof of concept and SWRM use cases}

As a proof of concept, sWRM was applied to Boulder County, Colorado. Weather data for this demonstration come from NOAA's HRRR weather product. The HRRR is an 18-h forecast updated hourly. Most output data have an hourly output resolution, with some fields available every $15 \mathrm{~min}$. The HRRR is chosen because of its high quality and its availability to the general public. The $3-\mathrm{km}$ resolution of the HRRR is insufficient for detailed trajectory modeling or path planning but may be useful for high-level mission planning over a relatively large area. Only the lowest four altitude layers of the HRRR are relevant to sUAS operations under Part 107 that take place below $400 \mathrm{ft}(120 \mathrm{~m})$ AGL. For the current demonstration, only the lowest layer of weather data ( $\sim 10 \mathrm{~m}$ AGL) and a single forecast hour is used, so results are those for just a snapshot of time and operations within about $30 \mathrm{~m}$ of the ground. Figures 4 and 5 show the HRRR mean wind speed and gusts, respectively, used as inputs to sWRM for this proof-of-concept demonstration. Temperature, humidity, turbulence, and wind shear data from the HRRR were also used in this study but for brevity are not plotted.

Structure density data used here are from the Global Human Built-Up and Settlement Extent (HBASE) dataset from Landsat (Wang et al. 2017). At each geographic grid point, this dataset gives the probability that HBASE is present with spatial resolution of $30 \mathrm{~m}$. This database does not provide "type" information about the HBASE that could be present at each grid point (roads, wood structures, glass buildings, building height, etc.). Databases with this level of detail may be available over specific areas, but the authors are unaware of a database with this level of detail for the CONUS. Future sUAS risk models could use more detailed datasets over the 


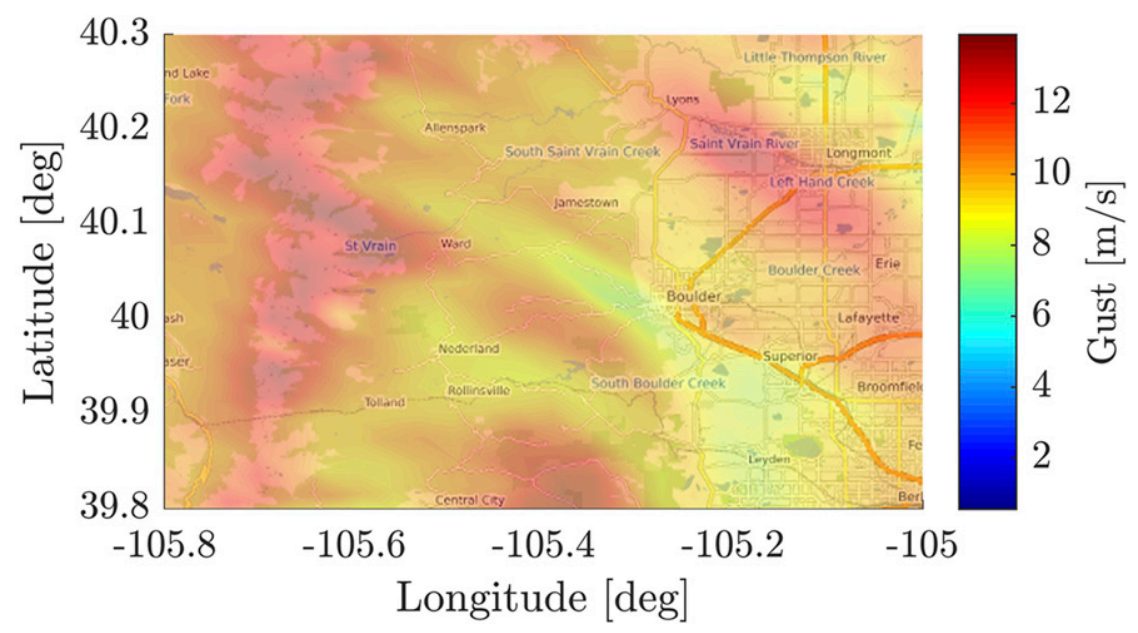

FIG. 5. Gust over Boulder County from HRRR forecast.

specific area being considered. Figure 6 shows the Boulder County HBASE from this Landsat dataset. The population density data used here come from the Gridded Population of the World, version 4 (GPWv4), dataset (Center for International Earth Science Information Network 2018) that provides an estimated number of persons per square kilometer on a 30 arc $\mathrm{s}$ grid $(\sim 1 \mathrm{~km}$ at equator). This dataset is based on where people permanently live. It does not provide information about the movement of people over time or whether or not there are coverings over people. While this dataset provides a good demonstration of estimating the risk to bystanders, future studies should incorporate dynamic models of person density that take into account changes due to the time of day, season of the year, special events, covering over people, etc. Local databases may be able to provide some of these higher-order details, and future studies should address how these added details will change the risk calculation. Figure 7 shows the Boulder County population density from the GPWv4 dataset.

The DJI Inspire 2 quadcopter sUAS is used for this proof of concept, and its properties are given in Table 7. Results from sWRM are shown in Figs. 8 and 9. Figure 8 demonstrates the primary goal of this technology: to provide weather-aware spatial risk maps for planning sUAS operations. The map shows where the risk is high (red), medium (yellow), or low (green). Figure 9 shows which hazard outcome has the highest risk. This information can help guide risk mitigation strategies for operators. The general trends seen in the figure are to be expected. Over populated areas (right side of the map), the risk is generally higher because of the increased likelihood of colliding with persons or structures. The risk decreases over rural to remote areas. However, there are several

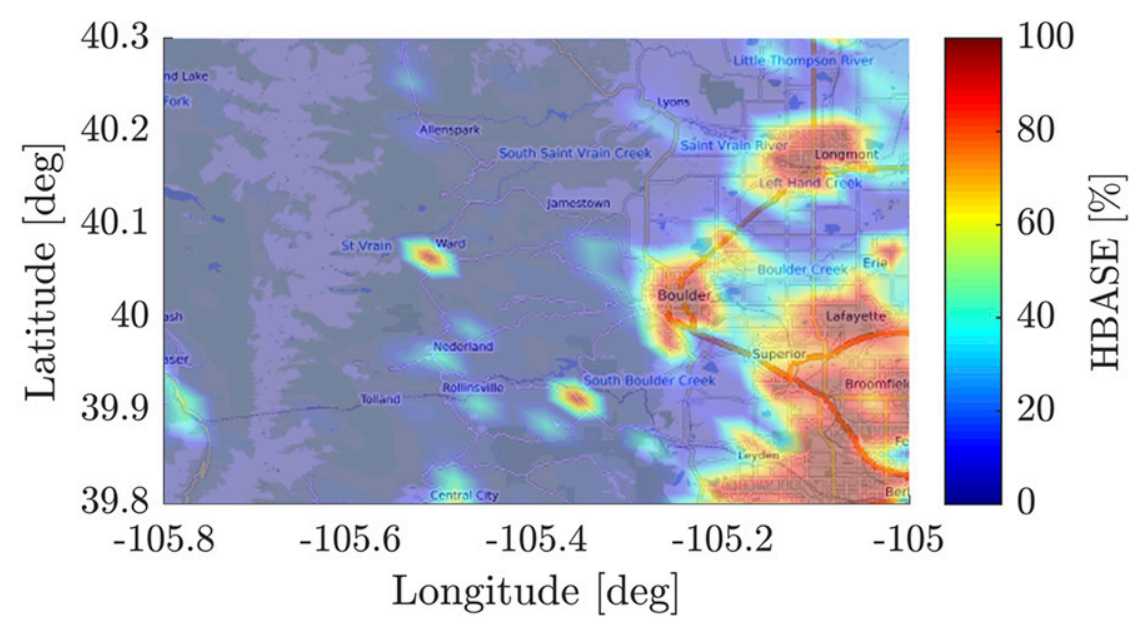

FIG. 6. Human Built-Up and Settlement Extent (HBASE) over Boulder County from Landsat. 


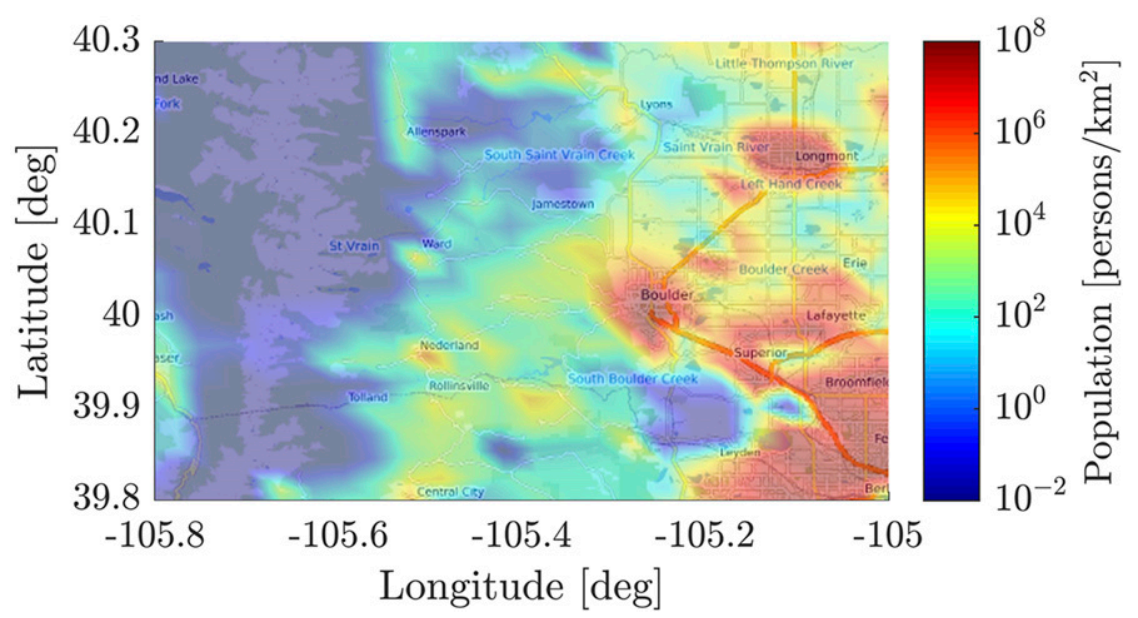

FIG. 7. Population density of Boulder County from GPWv4.

features the model produces that may not be so intuitive without such a systematic risk estimation tool.

The area over the continental divide (left side of the map) shows an increased risk for damage to aircraft and collision with person. In this area, the population and building density are both remote, so one may presume a low risk for collision with person. The medium risk calculated for collision with person is due to the high total wind speed in these areas (see Figs. 4 and 5), which causes the severity of collision with person to increase to a catastrophic severity level, where the risk can only be medium or high (see Fig. 1).

Harm to operator appears to be a prominent hazard on the right side of the map. This is due to particularly high temperatures, which were caused by a large longwave ridge that dominated the upper-level circulation over the CONUS during this time $\left(\sim 35^{\circ} \mathrm{C}\right)$ (National Centers for Environmental Information 2018). Part 107 currently requires VLOS between the operator and aircraft. This generally requires the operator to be outside, exposed to the elements. While it may be a conservative assessment, sWRM is assigning a medium risk for harm to operator because of the possibility of heatrelated incapacitation (Sheng et al. 2018).

In addition to this proof of concept, the sWRM framework has a number of possible use cases. The risk model framework presented can be used by the meteorological community for performing sensitivity studies to determine the most important weather types in different geographic areas. This information will help meteorologists tune high-resolution weather forecasts to produce accurate predictions for the weather types most relevant to sUAS.

The current framework could be expanded to estimate risk in real time. As mentioned in the introduction, some weather data could be provided by sensors on the UA. Real-time in situ weather data could allow sUAS to dynamically calculate their current risk state. Weather data inputs (wind, temperature, etc.) could come from sensors on the UA or nearby weather stations. Any weather type not directly sensed could be estimated or come from a forecast dataset. Other UA properties (altitude, speed, etc.) would come directly from the current UA state. Environment properties (structure and population density) could also be sensed if the sUAS is equipped with a camera and appropriate image processing software. Real-time dynamic risk assessment would be of great value for BVLOS operations and for urban operations where weather and environment inputs are highly variable. Enabling technologies for dynamic risk assessment are lowcost and accurate onboard weather sensing systems. Data from these onboard systems must be quickly processed to provide sUAS operators with timerelevant risk information.

TABLE 7. Properties of the DJI Inspire 2 sUAS used in the case study (asterisks indicate estimated values).

\begin{tabular}{lc}
\hline \multicolumn{1}{c}{ Property } & Value \\
\hline Aircraft mass $(\mathrm{kg})$ & 3.6 \\
Aircraft configuration & Multirotor \\
Max airspeed $\left(\mathrm{m} \mathrm{s}^{-1}\right)$ & 26 \\
Cruise airspeed $\left(\mathrm{m} \mathrm{s}^{-1}\right)$ & 10 \\
Cruise alt AGL $(\mathrm{m})$ & 10 \\
Automatic control & Yes \\
Disturbance rejection & Stock \\
Drag coef & 0.1 \\
Cross-sectional area ${ }^{*}\left(\mathrm{~m}^{2}\right)$ & 0.1 \\
Impact diam $^{*}(\mathrm{~m})$ & 0.2 \\
\hline
\end{tabular}




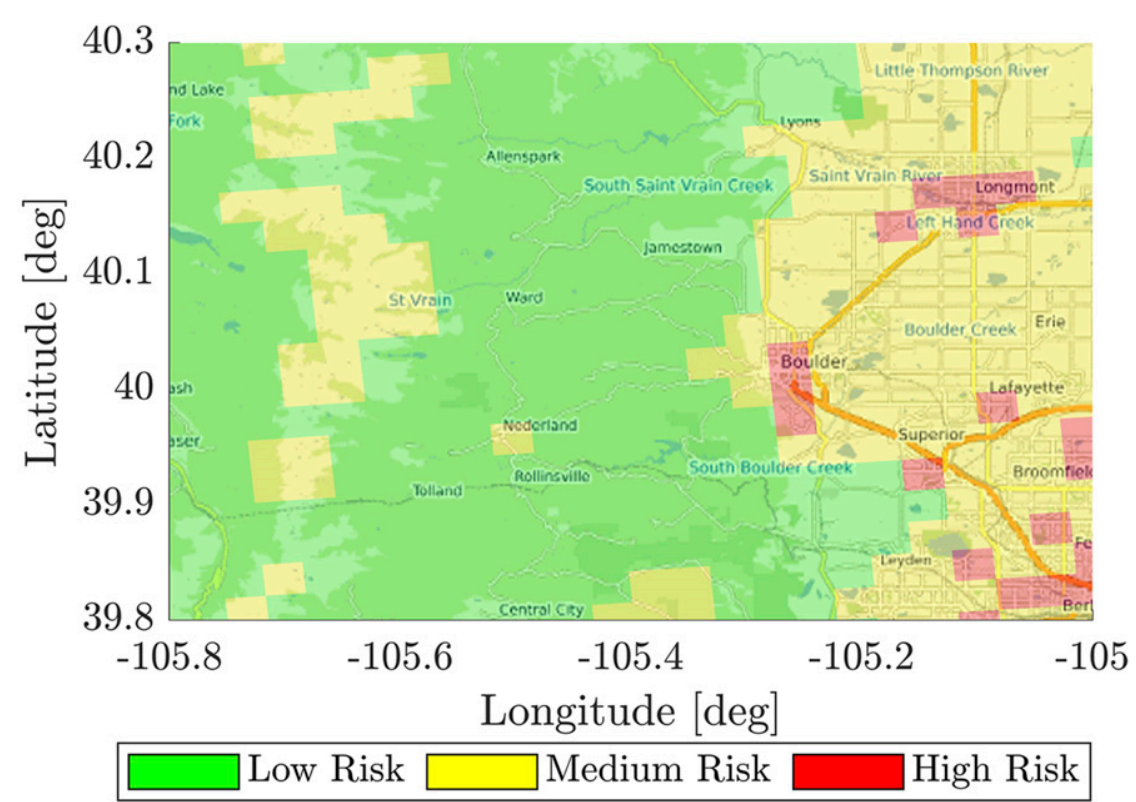

FIG. 8. Map of predicted sUAS risk level over Boulder County.

In addition to the aerospace and meteorological communities, comprehensive sUAS risk assessment technologies that include the impact of weather could be of great use for lawmakers, regulators, and insurance companies. The presented technology could be used for sensitivity studies investigating things such as the behavior of different aircraft configurations (e.g., fixed wing vs multirotor) in different weather environments (e.g., mountain vs plain regions or varying turbulence levels). This could help to guide region-specific and weather-aware regulations and insurance policies for sUAS.

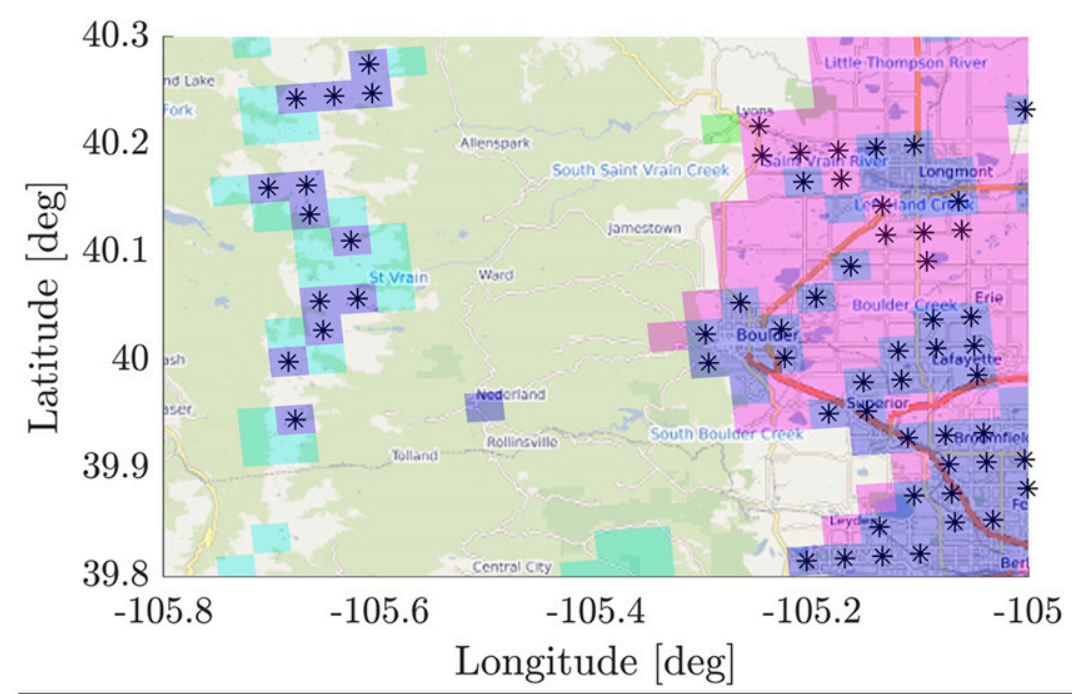

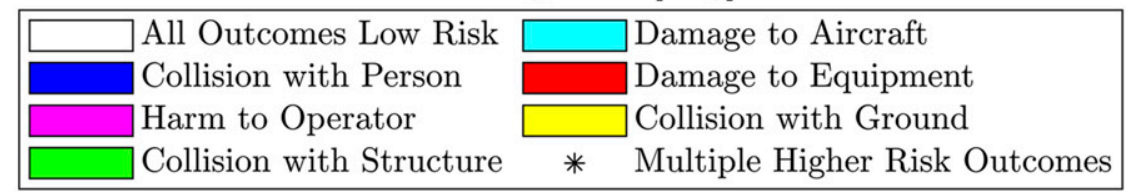

FIG. 9. Map showing the most hazardous outcome at different locations in the domain. Asterisks indicate regions for which there are at least two outcomes at the highest risk level. 


\section{Enabling research thrusts for operational sUAS weather risk assessment}

The development of sWRM highlights several research areas that must be addressed prior to the deployment of a fully operational weather hazard risk assessment tool; some enabling research thrusts are now discussed.

Many sUAS operations have a geographic footprint on the order of one square kilometer or less. The resolution of the HRRR, and other commonly available weather products, might be insufficient for risk assessment for these missions, especially where weather is highly variable in space and time, such as urban environments, requiring forecasts on smaller scales. Because of the general susceptibility of sUAS to turbulence and gusts, an important feature of these finescale forecasts is accurate near-surface turbulence and gust modeling, thus driving the research need for finescale, low-altitude atmospheric modeling.

A fundamental aspect of the risk calculation is quantifying the likelihood of an outcome. Outcomes from weather are highly dependent on uncertainty in the forecast. The BBN approach to quantifying likelihood can directly incorporate forecast uncertainty provided by the weather product. For example, if a weather product predicts a single value for turbulence at any given location, then the BBN interprets the probability of that turbulence level as $100 \%$. However, if the forecast produces a distribution of possible values, that distribution can be a direct input to the weather nodes of the BBN. This is illustrated in Fig. 3 with the relative gust, solar storm, and gust nodes. The Texas Tech WRF Ensemble Modeling System and the HRRR Ensemble (Ancell 2013; Dowell et al. 2018) are two weather products that include forecast uncertainty. As implied in their names, both of these products produce a measure of forecast uncertainty by running simulation ensembles with stochastic parameter perturbations. However, neither of these products have resolution less than $3 \mathrm{~km}$. Efficiently computed uncertainty statistics for highresolution forecasts is a meteorological and computational challenge and is an enabling technology for operational risk assessment tools.

A central feature of sWRM is the BBN used to estimate outcome likelihood. The accuracy of this network is dependent upon the accuracy of the parameters used in CPTs. Before this BBN can be used in an operational setting, these parameters must be derived from data. Extensive sUAS flight-report studies, especially for flights in varying weather conditions, are necessary to accurately estimate the CPT parameters. The general construction of the sWRM BBN provides an opportunity for the collection of large amounts of data for estimating these parameters.
Operations under Part 107 are assumed in the sWRM framework, so weather phenomena such as lightning or precipitation are currently not included since they are unlikely to occur with regulatory compliance. An operational weather risk model should either add the influence of non-Part 107 weather types or be accompanied by some auxiliary system to ensure an sUAS operator complies with the weather-related Part 107 rules.

\section{Conclusions}

Comprehensive safety risk management studies are needed for the safe integration of sUAS into the NAS. The sensitivity of sUAS to weather will be a factor in mission disruptions and accidents. The sWRM weather risk model framework quantifies risk to sUAS operations from adverse weather. The framework is based on FAA SRM guidelines and employs a Bayesian belief network to calculate hazard likelihood; hazard severity is calculated as a function of aircraft impact kinetic energy. A proof-of-concept sWRM example shows the usefulness of, and need for, comprehensive risk models that account for adverse weather conditions. The development of this model framework highlights several meteorological and aerospace research areas that must be addressed before an operational sUAS weather risk assessment tool can be deployed. Widely available finescale weather forecasting, including near-surface turbulence modeling, is necessary for risk assessment of specific sUAS operations. Extensive flight-report studies of sUAS in varying weather conditions are vital for accurately estimating parameters for Bayesian belief network conditional probability tables used in sWRM.

Acknowledgments. This material is based upon work supported by the National Science Foundation under Grant 1650468. The authors acknowledge James Pinto of NCAR RAL for his help in understanding and using weather forecast data, as well as initial discussions about the impact of weather on sUAS. The authors also acknowledge the reviewers whose feedback greatly improved this article.

\section{REFERENCES}

Ancel, E., F. M. Capristan, J. V. Foster, and R. C. Condotta, 2017: Real-time risk assessment framework for unmanned aircraft system (UAS) traffic management (UTM). 17th AIAA Aviation Technology, Integration, and Operations Conf., Denver, CO, American Institute of Aeronautics and Astronautics, AIAA 2017-3273, https://doi.org/10.2514/6.2017-3273.

Ancell, B. C., 2013: Nonlinear characteristics of ensemble perturbation evolution and their application to forecasting highimpact events. Wea. Forecasting, 28, 1353-1365, https://doi.org/ 10.1175/WAF-D-12-00090.1. 
Arterburn, D. R., C. T. Duling, and N. R. Goli, 2017: Ground collision severity standards for UAS operating in the National Airspace System (NAS). 17th AIAA Aviation Technology, Integration, and Operations Conf., Denver, $\mathrm{CO}$, American Institute of Aeronautics and Astronautics, AIAA 2017-3778, https://doi.org/10.2514/6.2017-3778.

Aviation Safety Information Analysis and Sharing, 2010: Weatherrelated aviation accident study. Federal Aviation Administration Tech. Rep., 71 pp., https://www.asias.faa.gov/i/studies/20032007weatherrelatedaviationaccidentstudy.pdf.

Baklanov, A. A., and Coauthors, 2011: The nature, theory, and modeling of atmospheric planetary boundary layers. Bull. Amer. Meteor. Soc., 92, 123-128, https://doi.org/10.1175/ 2010BAMS2797.1.

Barlow, J. F., 2014: Progress in observing and modelling the urban boundary layer. Urban Climate, 10, 216-240, https://doi.org/ 10.1016/j.uclim.2014.03.011.

Barr, L. C., R. L. Newman, E. Ancel, C. M. Belcastro, J. V. Foster, J. K. Evans, and D. H. Klyde, 2017: Preliminary risk assessment for small unmanned aircraft systems. 17th AIAA Aviation Technology, Integration, and Operations Conf., Denver, CO, American Institute of Aeronautics and Astronautics, AIAA 2017-3272, https://doi.org/10.2514/6.2017-3272.

Belcastro, C. M., R. L. Newman, J. K. Evans, D. H. Klyde, L. C. Barr, and E. Ancel, 2017: Hazards identification and analysis for unmanned aircraft system operations. 17th AIAA Aviation Technology, Integration, and Operations Conf., Denver, CO, American Institute of Aeronautics and Astronautics, AIAA 2017-3269, https://doi.org/10.2514/6.2017-3269.

Benjamin, S. G., and Coauthors, 2016: A North American hourly assimilation and model forecast cycle: The Rapid Refresh. Mon. Wea. Rev., 144, 1669-1694, https://doi.org/10.1175/ MWR-D-15-0242.1.

Bir, C., and D. C. Viano, 2004: Design and injury assessment criteria for blunt ballistic impacts. J. Trauma, 57, 1218-1224, https://doi.org/10.1097/01.TA.0000114066.77967.DE.

,$- \ldots$, and A. King, 2004: Development of biomechanical response corridors of the thorax to blunt ballistic impacts. J. Biomech., 37, 73-79, https://doi.org/10.1016/S0021-9290(03) 00238-0.

Bonin, T., P. Chilson, B. Zielke, and E. Fedorovich, 2013: Observations of the early evening boundary-layer transition using a small unmanned aerial system. Bound.-Layer Meteor., 146, 119-132, https://doi.org/10.1007/s10546-0129760-3.

Bradford, S., 2018: FAA UTM concept of operations-V1.0. Federal Aviation Administration Tech. Rep., 44 pp., https:// utm.arc.nasa.gov/docs/2018-UTM-ConOps-v1.0.pdf.

Breunig, J., J. Forman, S. Sayed, L. Audenaerd, A. Branch, and M. Hadjimichael, 2018: Modeling risk-based approach for small unmanned aircraft systems. 2018 Aviation Technology, Integration, and Operations Conf., Atlanta, GA, American Institute of Aeronautics and Astronautics, AIAA 2018-3349, https://doi.org/10.2514/6.2018-3349.

Bristol, T., 2019: Safety management system manual. Federal Aviation Administration Air Traffic Organization Tech. Rep., 128 pp., https://www.faa.gov/air-traffic/publications/media/ ATO-SMS-Manual.pdf.

Byun, S., J. Park, W. A. Appiah, M.-H. Ryou, and Y. M. Lee, 2017: The effects of humidity on the self-discharge properties of $\mathrm{Li}\left(\mathrm{Ni}_{1 / 3} \mathrm{Co}_{1 / 3} \mathrm{Mn}_{1 / 3}\right) \mathrm{O}_{2} /$ graphite and $\mathrm{LiCoO}_{2} /$ graphite lithiumion batteries during storage. RSC Adv., 7, 10 915-10921, https://doi.org/10.1039/C6RA28516C.
Campbell, S. E., D. A. Clark, and J. E. Evans, 2017a: Preliminary weather information gap analysis for UAS operations. MIT Lincoln Laboratory Tech. Rep., 126 pp., https://www.ll.mit.edu/ sites/default/files/publication/doc/2018-05/Campbell_2017_ATC437.pdf.

,-- , and $\longrightarrow, 2017 \mathrm{~b}$ : Preliminary UAS weather research roadmap. MIT Lincoln Laboratory Tech. Rep., 62 pp., https:// www.ll.mit.edu/sites/default/files/publication/doc/2018-05/ Campbell_2017_ATC-438.pdf.

Castagno, J., C. Ochoa, and E. Atkins, 2018: Comprehensive riskbased planning for small unmanned aircraft system rooftop landing. Int. Conf. on Unmanned Aircraft Systems, Dallas, TX, Institute of Electrical and Electronics Engineers, 1031-1040, https://doi.org/10.1109/ICUAS.2018.8453483.

Center for International Earth Science Information Network, 2018: Documentation for the Gridded Population of the World, version 4 (GPWv4), revision 11. NASA Socioeconomic Data and Applications Center, accessed 1 August 2019, https://doi.org/ 10.7927/H49C6VHW.

Clare, V. R., J. H. Lewis, A. P. Mickiewicz, and L. M. Sturdivan, 1975: Blunt trauma data correlation. Edgewood Arsenal Tech. Rep., 54 pp., https://apps.dtic.mil/docs/citations/ ADA012761.

Cornman, L. B., and W. N. Chan, 2017: Summary of a workshop on integrating weather into unmanned aerial system traffic management. Bull. Amer. Meteor. Soc., 98, ES257-ED259, https://doi.org/10.1175/BAMS-D-16-0284.1.

Dalamagkidis, K., K. P. Valavanis, and L. A. Piegl, 2008: Evaluating the risk of unmanned aircraft ground impacts. 16th Mediterranean Conf. on Control and Automation, Ajaccio, France, Institute of Electrical and Electronics Engineers, 709-716, https://doi.org/10.1109/MED.2008.4602249.

de Boer, G., and Coauthors, 2020: Development of community, capabilities and understanding through unmanned aircraftbased atmospheric research: The LAPSE-RATE campaign. Bull. Amer. Meteor. Soc., 101, E684-E699, https://doi.org/ 10.1175/BAMS-D-19-0050.1.

Denney, E., G. Pai, and M. Johnson, 2018: Towards a rigorous basis for specific operations risk assessment of UAS. IEEE/AIAA 37th Digital Avionics Systems Conf., London, United Kingdom, Institute of Electrical and Electronics Engineers, https://doi.org/ 10.1109/DASC.2018.8569475.

Dowell, D., C. Alexander, T. Alcott, and T. Ladwig, 2018: HRRR Ensemble (HRRRE) guidance 2018 HWT Spring Experiment. Earth System Research Laboratory Tech. Rep., 6 pp., https:// rapidrefresh.noaa.gov/hrrr/HRRRE/.

Duncan, J. S., 2016: Small unmanned aircraft systems (sUAS). U.S. Department of Transportation Rep. AC 107-2, 52 pp., https://www.faa.gov/documentlibrary/media/advisory_circular/ ac_107-2.pdf.

Elston, J. S., J. Roadman, M. Stachura, B. Argrow, A. Houston, and E. Frew, 2011: The tempest unmanned aircraft system for in situ observations of tornadic supercells: Design and VORTEX2 flight results. J. Field Rob., 28, 461-483, https:// doi.org/10.1002/rob.20394.

- , B. Argrow, M. Stachura, D. Weibel, D. Lawrence, and D. Pope, 2015: Overview of small fixed-wing unmanned aircraft for meteorological sampling. J. Atmos. Oceanic Technol., 32, 97-115, https://doi.org/10.1175/JTECH-D-13-00236.1.

Erdelj, M., E. Natalizio, K. R. Chowdhury, and I. F. Akyildiz, 2017: Help from the sky: Leveraging UAVs for disaster management. IEEE Pervasive Comput., 16, 24-32, https://doi.org/ 10.1109/MPRV.2017.11. 
Fenton, N., and M. Neil, 2013: Risk Assessment and Decision Analysis with Bayesian Networks. CRC Press, 660 pp.

Geipel, J., J. Link, W. Claupein, 2014: Combined spectral and spatial modeling of corn yield based on aerial images and crop surface models acquired with an unmanned aircraft system. Remote Sens., 6, 10335, https://doi.org/10.3390/RS61110335.

Glasheen, K., J. Pinto, M. Steiner, and E. Frew, 2019: Experimental assessment of local weather forecasts for small unmanned aircraft flight. Scitech 2019 Forum, Reston, VA, American Institute of Aeronautics and Astronautics, AIAA 2019-1193, https://doi.org/10.2514/6.2019-1193.

Guglieri, G., A. Lombardi, and G. Ristorto, 2015: Operation oriented path planning strategies for RPAS. Amer. J. Sci. Technol., 2, 321-328, http://www.aascit.org/journal/archive2? journalId $=902 \&$ paperId $=3358$.

Haddal, C. C., and J. Gertler, 2010: Homeland Security: Unmanned aerial vehicles and border surveillance. Congressional Research Service Tech. Rep., 10 pp., www.crs.gov.

Holtslag, A. A. M., and Coauthors, 2013: Stable atmospheric boundary layers and diurnal cycles: Challenges for weather and climate models. Bull. Amer. Meteor. Soc., 94, 1691-1706, https://doi.org/10.1175/BAMS-D-11-00187.1.

Houston, A. L., R. J. Laurence, T. W. Nichols, S. Waugh, B. Argrow, and C. L. Ziegler, 2016: Intercomparison of unmanned aircraftborne and mobile mesonet atmospheric sensors. J. Atmos. Oceanic Technol., 33, 1569-1582, https:// doi.org/10.1175/JTECH-D-15-0178.1.

Huerta, M. P., 2012: Safety risk management policy. Federal Aviation Administration Tech. Rep., 33 pp., https://www.faa.gov/ documentLibrary/media/Order/FAA_Order_8040.4B.pdf.

Hunt, E. R., W. D. Hively, S. J. Fujikawa, D. S. Linden, C. S. T. Daughtry, and G. W. McCarty, 2010: Acquisition of NIR-greenblue digital photographs from unmanned aircraft for crop monitoring. Remote Sens., 2, 290-305, https://doi.org/10.3390/rs2010290.

Kim, S. H., 2019: Third-party risk of mid-air collision between small unmanned aircraft systems. AIAA Aviation Forum, Dallas, TX, American Institute of Aeronautics and Astronautics, AIAA 2019-3052, https://doi.org/10.2514/6.2019-3052.

Koch, S. E., M. Fengler, P. B. Chilson, K. L. Elmore, B. Argrow, D. L. Andra, and T. Lindley, 2018: On the use of unmanned aircraft for sampling mesoscale phenomena in the preconvective boundary layer. J. Atmos. Oceanic Technol., 35, 2265-2288, https://doi.org/10.1175/JTECH-D-18-0101.1.

Krishnakumar, K., P. Kopardekar, C. Ippolito, J. E. Melton, V. Stepanyan, S. Sankararaman, and B. Nikaido, 2017: Safe autonomous flight environment (SAFE50) for the notional last " $50 \mathrm{ft}$ " of operation of " $55 \mathrm{lb}$ " class of UAS. AIAA Information Systems-AIAAInfotech@ Aerospace, Grapevine, TX, American Institute of Aeronautics and Astronautics, AIAA 2017-0445, https://doi.org/10.2514/6.2017-0445.

La Cour-Harbo, A., 2019: Quantifying risk of ground impact fatalities for small unmanned aircraft. J. Intell. Rob. Syst., 93, 367-384, https://doi.org/10.1007/s10846-018-0853-1.

Lee, G.-J., D. Muñoz-Esparza, C. Yi, and H. J. Choe, 2019: Application of the cell perturbation method to large-eddy simulations of a real urban area. J. Appl. Meteor. Climatol., 58, 1125-1139, https://doi.org/10.1175/JAMC-D-18-0185.1.

Li, X.-X., D. Y. C. Leung, C.-H. Liu, and K. M. Lam, 2008: Physical modeling of flow field inside urban street canyons. J. Appl. Meteor. Climatol., 47, 2058-2067, https://doi.org/ 10.1175/2007JAMC1815.1.

Lukacs, M., and D. Bhadra, 2017: FAA Aerospace Forecast: Fiscal years 2018-2038. Federal Aviation Administration
Tech. Rep., 101 pp., https://www.faa.gov/data_research/ aviation/aerospace_forecasts/media/FY2018-38_FAA_Aerospace_ Forecast.pdf.

Lundby, T., M. P. Christiansen, and K. Jensen, 2019: Towards a weather analysis software framework to improve UAS operational safety. Int. Conf. on Unmanned Aircraft Systems, Atlanta, GA, Institute of Electrical and Electronics Engineers, 13721380, https://doi.org/10.1109/icuas.2019.8798271.

Luxhøj, J. T., 2013: Predictive analytics for modeling UAS safety risk. SAE Int. J. Aerosp., 6, 128-138, https://doi.org/10.4271/ 2013-01-2104.

2015: A socio-technical model for analyzing safety risk of unmanned aircraft systems (UAS): An application to precision agriculture. Procedia Manuf., 3, 928-935, https://doi.org/ 10.1016/j.promfg.2015.07.140.

—_, and A. Öztekin, 2009: A regulatory-based approach to safety analysis of unmanned aircraft systems. Engineering Psychology and Cognitive Ergonomics, D. Harris, Ed., Vol. 5639, Springer, 564-573, https://doi.org/10.1007/978-3-642-02728-4-60.

Magister, T., 2010: The small unmanned aircraft blunt criterion based injury potential estimation. Saf. Sci., 48, 1313-1320, https://doi.org/10.1016/j.ssci.2010.04.012.

Mahrt, L., 2014: Stably stratified atmospheric boundary layers. Annu. Rev. Fluid Mech., 46, 23-45, https://doi.org/10.1146/ annurev-fluid-010313-141354.

Martin, G., 2019: Press release-U.S. Transportation Secretary Elaine L. Chao announces FAA certification of commercial package delivery. Federal Aviation Administration, https://www.faa.gov/ news/press_releases/news_story.cfm?newsId $=23554$.

Mathew, N., S. L. Smith, and S. L. Waslander, 2015: Planning paths for package delivery in heterogeneous multirobot teams. IEEE Trans. Autom. Sci. Eng., 12, 1298-1308, https://doi.org/ 10.1109/TASE.2015.2461213.

Murray, C. C., and A. G. Chu, 2015: The flying sidekick traveling salesman problem: Optimization of drone-assisted parcel delivery. Transp. Res., 54, 86-109, https://doi.org/10.1016/ j.trc.2015.03.005.

National Academies of Sciences, Engineering, and Medicine, 2018: Assessing the Risks of Integrating Unmanned Aircraft Systems (UAS) into the National Airspace System. National Academies Press, 78 pp., https://www.nap.edu/catalog/25143/assessingthe-risks-of-integrating-unmanned-aircraft-systems-uas-intothe-national-airspace-system.

National Centers for Environmental Information, 2018: State of the climate: Synoptic discussion for June 2018. National Oceanic and Atmospheric Administration, https://www.ncdc.noaa.gov/ sotc/synoptic/201806.

Nolan, P. J., and Coauthors, 2018: Coordinated unmanned aircraft system (UAS) and ground-based weather measurements to predict Lagrangian coherent structures (LCSs). Sensors, 18, 4448, https://doi.org/10.3390/s18124448.

Öztekin, A., and J. Luxhøj, 2008: Hazard, safety risk and uncertainty modeling of the integration of unmanned aircraft systems into the National Airspace. 26th Int. Congress of the Aeronautical Sciences, Anchorage, AK, International Council of the Aeronautical Sciences, 347-356, http://icas.org/icasarchive/icas2008/papers/062.pdf.

Primatesta, S., G. Guglieri, and A. Rizzo, 2019: A risk-aware path planning strategy for UAVs in urban environments. J. Intell. Rob. Syst., 95, 629-643, https://doi.org/10.1007/s10846-018-0924-3.

, A. Rizzo, and A. La Cour-Harbo, 2020: Ground risk map for unmanned aircraft in urban environments. J. Intell. Rob. Syst., 97, 489-509, https://doi.org/10.1007/S10846-019-01015-Z. 
Ranquist, E., M. Steiner, and B. Argrow, 2017: Exploring the range of weather impacts on UAS operations. 18th Conf. on Aviation, Range, and Aerospace Meteorology, Seattle, WA, Amer. Meteor. Soc., J3.1, https://ams.confex.com/ams/97Annual/webprogram/ Paper309274.html.

Roseman, C. A., B. M. Argrow, and J. O. Pinto, 2019: Targeted weather forecasts for small unmanned aircraft systems. 19th Conf. on Aviation, Range, and Aerospace Meteorology, Phoenix, AZ, Amer. Meteor. Soc., 1.4, https://ams.confex.com/ams/ 2019Annual/webprogram/Paper351492.html.

Schultz, P., and M. K. Politovich, 1992: Toward the improvement of aircraft-icing forecasts for the continental United States. Wea. Forecasting, 7, 491-500, https://doi.org/10.1175/15200434(1992)007<0491:TTIOAI >2.0.CO;2.

Sheng, R., and Coauthors, 2018: Does hot weather affect workrelated injury? A case-crossover study in Guangzhou, China. Int. J. Hyg. Environ. Health, 221, 423-428, https://doi.org/ 10.1016/j.ijheh.2018.01.005.

Shim, J., R. Kostecki, T. Richardson, X. Song, and K. A. Striebel, 2002: Electrochemical analysis for cycle performance and capacity fading of a lithium-ion battery cycled at elevated temperature. J. Power Sources, 112, 222-230, https://doi.org/ 10.1016/S0378-7753(02)00363-4.

Skamarock, W. C., and Coauthors, 2019: A description of the Advanced Research WRF Model version 4. NCAR Tech. Rep. NCAR/TN-556+STR, 162 pp., https://doi.org/10.5065/ $1 \mathrm{dfh}-6 \mathrm{p} 97$.

Sturdivan, L. M., D. C. Viano, and H. R. Champion, 2004: Analysis of injury criteria to assess chest and abdominal injury risks in blunt and ballistic impacts. J. Trauma, 56, 651-663, https:// doi.org/10.1097/01.TA.0000074108.36517.D4.
Thiels, C. A., J. M. Aho, S. P. Zietlow, and D. H. Jenkins, 2015: Use of unmanned aerial vehicles for medical product transport. Air Med. J., 34, 104-108, https://doi.org/10.1016/j.amj.2014.10.011.

Tuna, G., B. Nefzi, and G. Conte, 2014: Unmanned aerial vehicle-aided communications system for disaster recovery. J. Network Comput. Appl., 41, 27-36, https://doi.org/ 10.1016/j.jnca.2013.10.002.

Wackwitz, K., and H. Boedecker, 2015: Safety risk assessment for UAV operation. Drone Industry Insights Tech. Rep., 16 pp., https:// miningquiz.com/pdf/Drone_Safety/Safety-Risk-Assessmentfor-UAV-Operation-Rev.-1.1.compressed.pdf.

Wang, P., C. Huang, E. C. Brown de Colstoun, J. C. Tilton, and B. Tan, 2017: Documentation for the Global Human Built-Up and Settlement Extent (HBASE) dataset from Landsat. NASA Socioeconomic Data and Applications Center, accessed 14 August 2019, https://doi.org/10.7927/H4DN434S.

Washington, A., R. A. Clothier, and J. Silva, 2017a: A review of unmanned aircraft system ground risk models. Prog. Aerosp. Sci., 95, 24-44, https://doi.org/10.1016/j.paerosci.2017.10.001.

$\longrightarrow,-$, and B. P. Williams, 2017b: A Bayesian approach to system safety assessment and compliance assessment for unmanned aircraft systems. J. Air Transp. Manage., 62, 18-33, https://doi.org/10.1016/j.jairtraman.2017.02.003.

,-- N. Neogi, J. Silva, K. Hayhurst, and B. Williams, 2019: Adoption of a Bayesian belief network for the system safety assessment of remotely piloted aircraft systems. Saf. Sci., 118, 654-673, https://doi.org/10.1016/j.ssci.2019.04.040.

Zhang, C., J. M. Kovacs, C. Zhang, and J. M. Kovacs, 2012: The application of small unmanned aerial systems for precision agriculture: A review. Precis. Agric., 13, 693-712, https:// doi.org/10.1007/s11119-012-9274-5. 